\title{
Detection and Correction of Under-/Overexposed Optical Soundtracks by Coupling Image and Audio Signal Processing
}

\author{
Jonathan Taquet, ${ }^{1}$ Bernard Besserer, ${ }^{1}$ Abdelali Hassaine, ${ }^{2}$ and Etienne Decenciere ${ }^{2}$ \\ ${ }^{1}$ Laboratoire Informatique, Image, Interaction, Université de La Rochelle, 17042 La Rochelle, France \\ ${ }^{2}$ Centre de Morphologie Mathématique, Ecole Nationale Supérieure des Mines de Paris, 77305 Fontainebleau, France
}

Correspondence should be addressed to Bernard Besserer, bernard.besserer@univ-lr.fr

Received 2 October 2007; Revised 15 June 2008; Accepted 26 June 2008

Recommended by Anil Kokaram

\begin{abstract}
Film restoration using image processing, has been an active research field during the last years. However, the restoration of the soundtrack has been mainly performed in the sound domain, using signal processing methods, despite the fact that it is recorded as a continuous image between the images of the film and the perforations. While the very few published approaches focus on removing dust particles or concealing larger corrupted areas, no published works are devoted to the restoration of soundtracks degraded by substantial underexposure or overexposure. Digital restoration of optical soundtracks is an unexploited application field and, besides, scientifically rich, because it allows mixing both image and signal processing approaches. After introducing the principles of optical soundtrack recording and playback, this contribution focuses on our first approaches to detect and cancel the effects of under and overexposure. We intentionally choose to get a quantification of the effect of bad exposure in the 1D audio signal domain instead of 2D image domain. Our measurement is sent as feedback value to an image processing stage where the correction takes place, building up a "digital image and audio signal" closed loop processing. The approach is validated on both simulated alterations and real data.
\end{abstract}

Copyright (C) 2008 Jonathan Taquet et al. This is an open access article distributed under the Creative Commons Attribution License, which permits unrestricted use, distribution, and reproduction in any medium, provided the original work is properly cited.

\section{INTRODUCTION}

A general introduction should be useful, because very few people are familiar with optical soundtracks. In fact, most people do not even know how sound is carried for theatrical release prints, the most popular thoughts on this issue would be a separate accompanying material for the sound (which is true for Digital Theater System (DTS). In fact, over almost 80 years, the sound is carried among the pictures on the film stock itself, as an optical track, for both analog sound and modern digital sound (Dolby Digital or Sony Dynamic Digital Sound (SDDS). We focus in this paper on analog soundtracks, used from the thirties until today, and still present on release copies as backup when the reading of digital data fails (see Figure 1).

Looking at facts and compared to up-to-date technology, analog optical sound has a narrow dynamic range, as well as a limited frequency response. But early sound (from the thirties) was intelligible, often pleasant to listen to (from the fifties up, the technology became mature), showed incred- ible interoperability between evolving standards, and the analog soundtrack is somehow robust against impairments. Optical sound recording has indeed an interesting and rich history [1-4]. Motion pictures have historically employed several types of optical soundtracks, ranging from variable density (VD) to stereophonic variable area (VA) tracks (see Figure 2). For many years, the standard industry practice for the $35 \mathrm{~mm}$ theatrical release format has been the variable area optical soundtrack, called The standard Academy Optical Mono track and introduced by "the Academy of Motion Picture Arts and Sciences," (ca. 1938). Between the sprocket holes and the picture, a $1 / 10$ inch $(\mathrm{ca} .3 \mathrm{~mm}$ ) is dedicated to the optical soundtrack.

In general, sound is recorded on the film by exposing this area to a source of light in an optical recorder. For VD soundtracks, the light intensity of the recorder is modulated and the film density, after processing, goes through varying shades of grey according to the exposure. For VA soundtracks, the geometry is modulated (width of exposed area), and the track comprises a portion which is essentially 


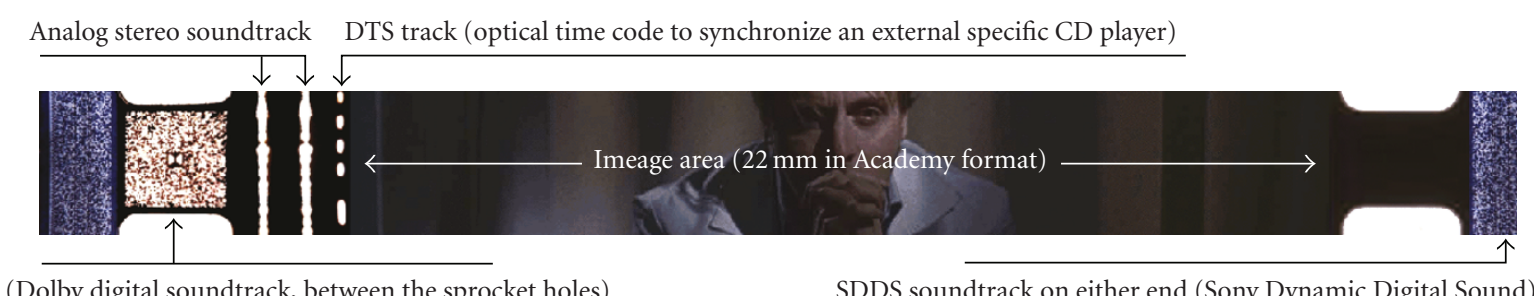

(Dolby digital soundtrack, between the sprocket holes)

SDDS soundtrack on either end (Sony Dynamic Digital Sound)

FIGURE 1: $35 \mathrm{~mm}$ film strip showing modern digital soundtracks among the analog VA soundtrack.

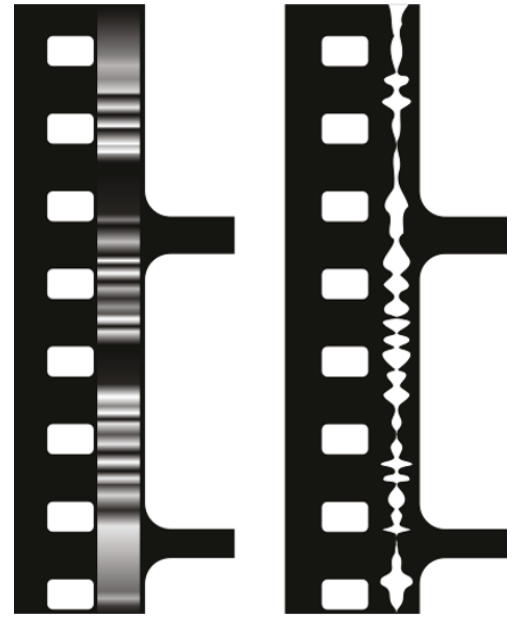

FIGURE 2: Left: variable density; right: variable area/fixed density.

opaque and a portion which is left essentially transparent, the ratio between the two portions being proportional to the instantaneous amplitude of the sound signal being recorded. The reading of the soundtrack consists in the inverted process. A light beam is projected through a slit, then through the film, which continuously streams and, therefore, modulates the light, while a photoelectric device picks up the amount of light and feeds the amplifier stage, as illustrated in Figure 3. Note that the same pickup head is able to read VA or VD tracks (in both cases, the amount of light varies) and stereo tracks can be read on a monopickup head, the light going through the left track is simply summed to the light going to the right track (optical mixing).

At reading, the VD process caused an important background noise, due to film grain and dust spots: every dust particle caused a variation of the intensity. The VA process is much more robust with respect to dust on the dark portions (black over black). This is one of the reasons the VD process was replaced by the VA process.

For the film industry, the standardization of sound reproduction has always been a necessity: the sound produced by the different studios, as well as its playback in different theatres, should be similar. Therefore, the sound system of a motion-picture theatre was divided into two parts- the A-chain (sound recording and playback) and the B-chain (amplifiers, loudspeakers, acoustics). For the A-chain, the

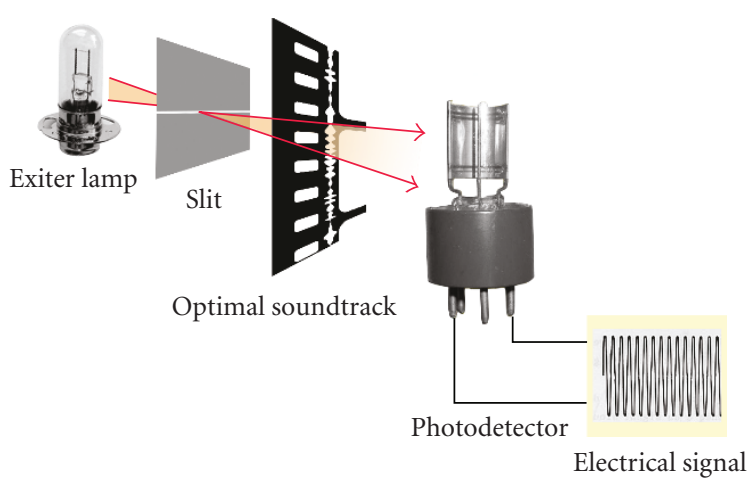

FIGURE 3: The reproduction process of a VA optical soundtrack.

oldest standard response curve is the A-Curve (Standard Electrical Characteristic of 1938, also called Academy Curve) [5]. The Academy Curve is flat from $100 \mathrm{~Hz}$ to $1.6 \mathrm{kHz}$ and falls rapidly beyond these limits, removing frequencies above $8 \mathrm{kHz}$ to avoid hiss. From the 1970's, this standard has needed an update and in 1984, a new SMPTE standard was published to formalize the new standard, named the XCurve for eXtended range curve (ANSI-SMPTE 202M and ISO2969). The X-Curve response is flat up to $2 \mathrm{kHz}$ then falls $3 \mathrm{~dB}$ per octave to $10 \mathrm{kHz}$, above which it falls at $6 \mathrm{~dB}$ per octave, as illustrated in Figure 4.

Nowadays, a bandwidth of $20 \mathrm{~Hz}$ to $14 \mathrm{kHz}$ is given for a modern optical recorder (Westrex/Nuoptix). The spatial resolution of the film stock used for optical soundtracks (Kodak 2302) is about 100 lines per $\mathrm{mm}$. Since a $35 \mathrm{~mm}$ film travels at $456 \mathrm{~mm}$ per second, the maximum "bandwidth" of a film itself as analog optical carrier does not exceed $22 \mathrm{kHz}$.

For the following work, the optical sound is oversampled at $48 \mathrm{kHz}$ by a line-scan camera, fitted with a reversemount Scheider-Kreuznach macrolens. The film stock is illuminated by a fibre optic line light guide (see Figure 5). The size of the resulting image is $48000 \times 512$ pixels for a second of sound. The rather poor line resolution is compensated by a 10 to 12 bits/pixels dynamics to capture precisely the luminance levels along the transition edges of the VA modulation. A specific scanner has been built around a reformed sepmag player (a device able to read sound recorded as separate magnetic tapes (magnetic coated $35 \mathrm{~mm}$ or $16 \mathrm{~mm}$ film stock)) in order to start a large-scale acquisition and restoration campaign and to validate the method for a very broad set of problems. 


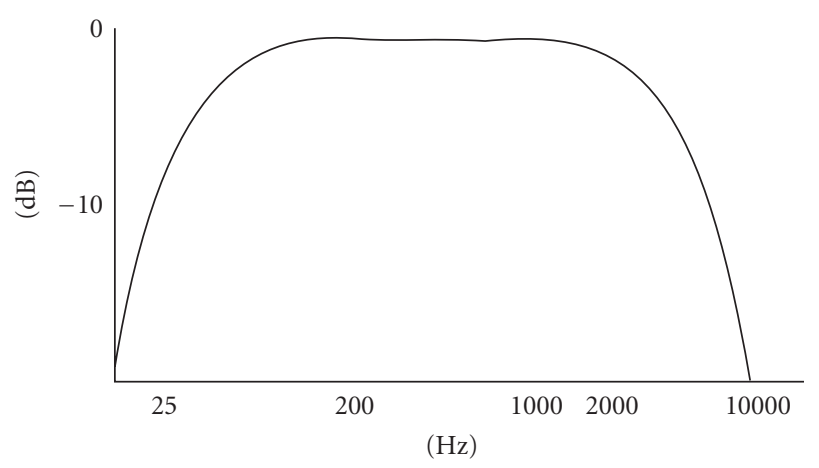

(a)

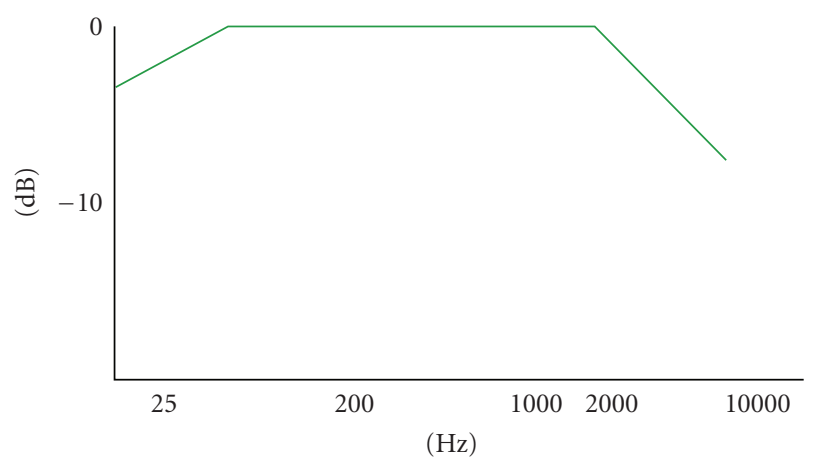

(b)

Figure 4: (a): bandwidth according to the A-curve. (b): bandwidth according to X-curve.

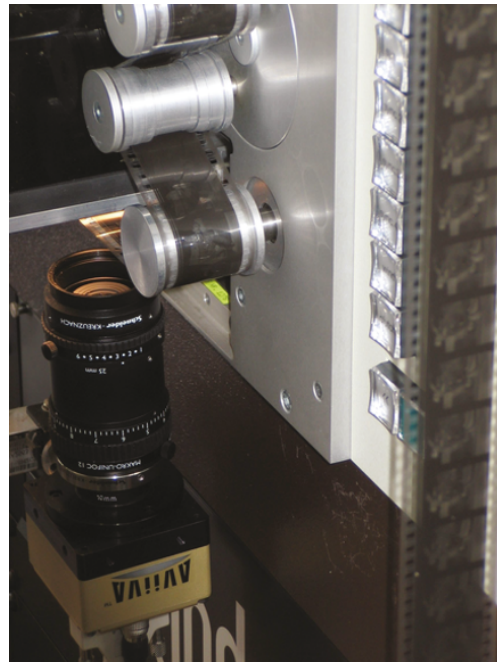

FIGURE 5: Close shot of our specific scanner, showing the line-scan camera and macrolens.

\section{OPTICAL SOUNDTRACKS ALTERATIONS}

Unfortunately, the optical soundtrack undergoes the same type of degradations as the image of the film (dust, scratches). Given that they are located close to the film stock edge, soundtracks are sometimes degraded by abrasion in the neighbourhood of the perforations or by fungus or mould attacking the film on an important surface. An example of corrupted soundtrack is shown in Figure 6.

Classically, sound processing and restoration are performed only after the transformation of the optical information into acoustic electric signal (see Figure 7). Impulsive impairments are easy to conceal in the 1-D signal domain, but the presence of large area degradation or repetitive defects on the soundtrack introduces distortions that are delicate to correct after the transformation: as powerful as they are, digital audio processing systems cannot make the difference between some audio artifacts caused by the degradation of the optical soundtrack, and some sounds present in the original soundtrack.
There are only few references in the literature on this topic. In 1999, Streule [6] proposed a soundtrack restoration method using digital image processing tools. He proposes a complete system, going from the soundtrack digitization, up to the generation of the corresponding audio file. Concerning the restoration, Streule only treats defects caused by dust. The proposed technique is mainly based on the soundtrack symmetry.

Richter et al. proposed in [7] a method of impairments localization in multiple double-sided variable area soundtracks, but they do not treat the correction of these impairments. This method eliminates low frequencies in Fourier Space, which correspond to small defects in the original image, and after a binarization, the remaining faults are sufficiently large to be easily detected. The same authors published also a paper about variable density soundtrack restoration [8].

Spots detection is also used by Kuiper in $[9,10]$. The spots being lighter than other parts of the image, a threshold isolates them. A succession of morphological operations is then applied for a better spot localization and for the removal of the isolated pixels. Unfortunately, in most cases, the spots are not lighter than the other parts of the image. For that reason, this method cannot be always used.

Valenzuela appears as inventor of several patents on soundtrack scanning and restoration. He proposes a short description of his technique in [11]. The restoration is very simple, and is based on median filters and erosions. It can only deal with the smallest defects.

To the extent of our knowledge, nothing has been published on the restoration of incorrectly exposed optical soundtracks.

None of the previous techniques would allow a satisfactory restoration of moderately to severely damaged soundtracks. This was one of the major reasons to start in 2005 a research program called RESONANCES, mainly aimed at restoration of optical soundtracks in the "image domain". Removing dust, scratches, and other defects is one of the aims of the project. An advanced image processing method has been developed in order to remove defects and restore the track symmetry [12]. A real-time dust-busting algorithm for VA soundtracks is also under development. 


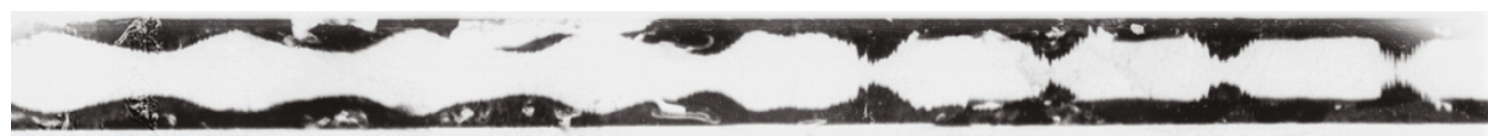

Figure 6: A heavily corrupted soundtrack (fungus or mould).

However, as stated before, this contribution focuses on the correction of over- and underexposed soundtracks. We can, therefore, hereafter assume that we deal with clean and symmetric samples.

\subsection{Underexposure and overexposure}

As for the image part of a movie, the optical soundtrack undergoes several copies, from the masterized soundtrack photographed by the optical recorder to the final print. Therefore, density control is important and the exposure should be set to use the straight-line portion (linear response) of the $\mathrm{H} \& \mathrm{D}$ curve (density versus exposure) on the original negative, as well as on intermediate and final prints. The film stock used and the parameters of the development process (temperature, use of fresh or used chemicals, etc.) influence also film density. The quality control for this production chain was of great importance for variable density soundtracks and hard to manage, and this is another reason for the demise of VD tracks. VA tracks are more tolerant to exposure and development conditions, since the pattern to be reproduced is more or less binary (transparent track, opaque surroundings). However, under certain conditions, bad exposure can affect significantly the VA track due to image spread (or flare) and the S-shaped response of the film. Suppose a small, sharply focused spot of light is exposed on a piece of film. After processing, the developed image is likely to be larger than the spot of light originally imaged on the film. In present day processing, according to the fact that negative films will tolerate overexposure to a greater degree than underexposure, and that more image spread happens in the print stock than in the negative stock, one has to greatly overexpose the negative to intentionally get image spread to cancel out the spread in the print. The crossmodulation test helps the labs technician to set correct exposure parameters, read more about this procedure in the appendix.

The distortion level induced by under-/overexposure is frequency dependant: the image shape does not change significantly for low-frequency signals (under $1 \mathrm{kHz}$ ). The image spread introduces first a desymmetrization of the signal and generates even harmonics as frequency increases above 2 or $3 \mathrm{kHz}$. At higher frequencies, the shape of the signal is altered, introducing moreover odd harmonics (Figure 10). If the frequency is above ca. $5 \mathrm{kHz}$, a pure sinusoidal wave takes on a sharper, more saw tooth shape, either on the inner side (underexposure) or the outer side (overexposure), as shown in Figure 8.

While listening, voice is mainly affected, especially the sibilants; but such distortion is hardly noticeable for music (especially music which is naturally rich in harmonics or partials, such as brass instruments).
On pure frequency signals, the effects of the overexposure are the same ones as those of the underexposure (with a phase shift of $\pi$ ).

It seems to be very hard and complex for an arbitrary 1D audio signal to distinguish between distortion introduced by overexposure from the distortion introduced by underexposure. Accordingly, and for the following reasons, we decide not to investigate this topic:

(1) separating overexposure from underexposure can be easily done in 2D image processing of the optical representation of the soundtrack;

(2) for our closed-loop approach (Figure 17), the sign of the feedback signal will be manually set by the operator.

\subsection{Simulation of optical soundtrack processing chain}

The physical phenomenon which causes the over-/underexposer is well known, and can be fairly accurately modelled in the image domain. We have, therefore, built an exposure simulator which deals with the optical representation of the soundtrack as $2 \mathrm{D}$ image and simulates the image spread. We designed a framework under MATLAB with a suitable user interface, illustrated in Figure 9, allowing us to calculate the following steps.

\section{Converting a WAVE PCM sound to its (perfect) optical representation}

The dynamic of the WAV samples is reduced to 256 steps. Each sample directly generates a binary image line (the width of the white area is in the range [0..512] due to the symmetric nature of the optical recording), and the output image is antialiased.

\section{Simulate the image spread}

We first convolve the image by a $2 \mathrm{D}$ gaussian kernel (a $2 \mathrm{D}$ squared cardinal sine filter can be selected as well, often used to model the point spread function in astronomy imagery). The resulting grey-levels are matched against a S-shaped (sigmoid) lookup table, roughly simulating the film transfer function.

\section{Convert the optical representation back to WAVE PCM sound}

The photocell integration is simulated for each line, luminosity of the pixels are summed up, the result is normalized to fit the WAVE dynamic range, and a high-pass filter is used to remove the DC component, as the decoupling capacitor does between the optical pickup head and the amplifier stage. 


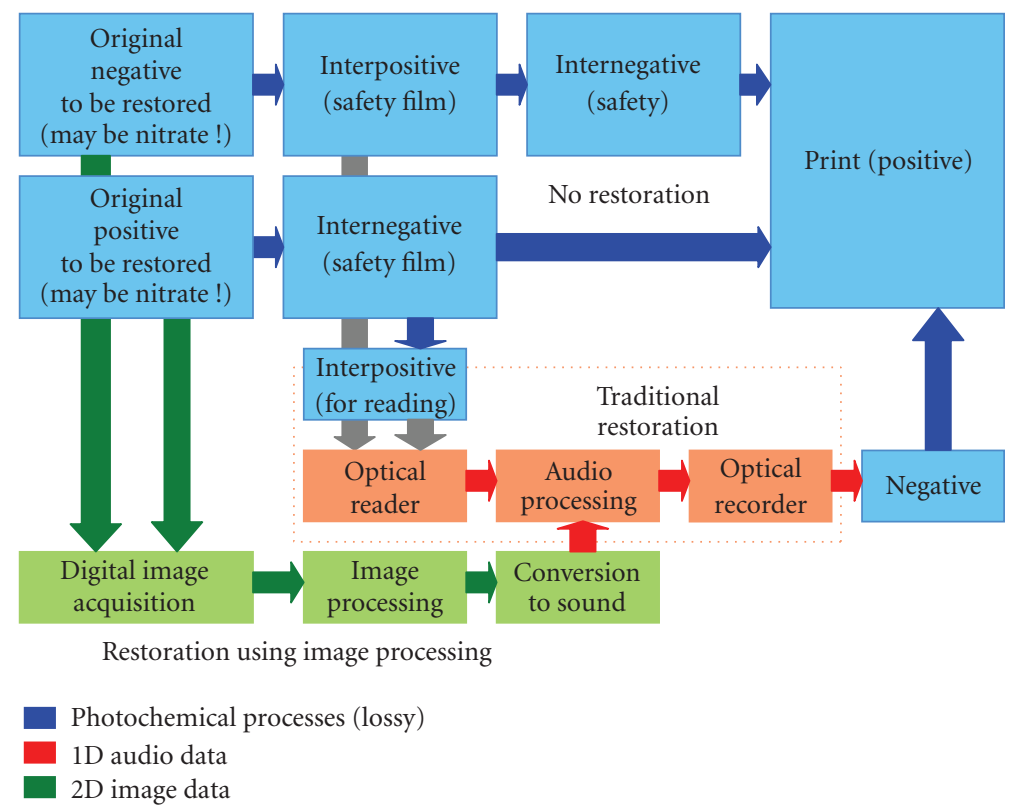

FIGURE 7: If the film to be restored is a positive, it may result from several intermediates-possibly including bad exposures. Nitrate film stock is often first copied on safety stock. Since a traditional optical pickup head cannot directly read negative, an interpositive is first printed. Digital processing can avoid such additional copy processes by digitizing the negative directly.

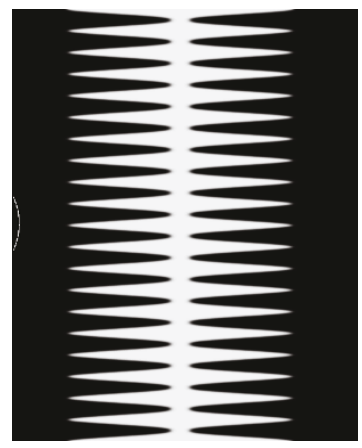

(a)

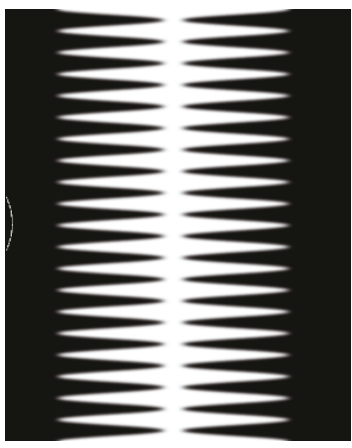

(b)

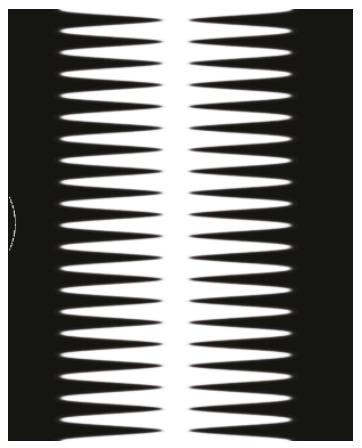

(c)

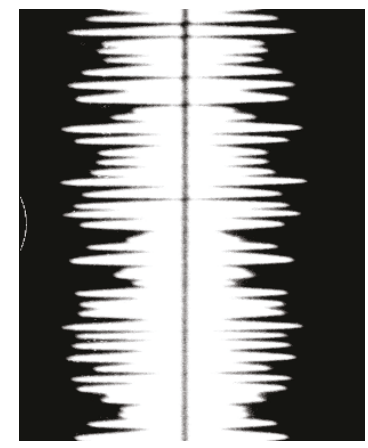

(d)

FiguRE 8: Test tone underexposed (a), correctly exposed (b), overexposed (c), and a real sound showing underexposure (d).

To check our simulation, we generate a sweep signal (sine wave, from $50 \mathrm{~Hz}$ to $10 \mathrm{kHz}$ ). After a simulated overexposure, the output spectrogram is shown in Figure 10.

\section{RESTORING UNDEREXPOSED AND OVEREXPOSED OPTICAL SOUNDTRACKS}

Restoring an ancient movie is a delicate task, and the curator's first step is to collect available film copies from several film archives, and keep the qualitative best parts. The optical soundtrack quality within the selected parts may range from correctly exposed print releases up to severely under/overexposed negatives. So, beside dust-busting-, symmetry enforcement-, and image-processing-related restoration of the optical soundtrack, we should be able to detect and correct possible under-/overexposure to level off the quality of the output soundtrack.

The restoration of the under-/overexposed soundtracks with image processing operators seems to be a promising strategy. Mathematical morphology [13] offers operators which are well adapted for dealing with this sort of geometrical problem.

The 1D audio curve itself can figure the boundary for a binary, image-like representation in a 2D space (amplitude, time), where the area "under the curve" is black (object) and "over the curve" is white (background), and, therefore, morphological operators can be applied on this dataset. However, since the problem of over-/underexposure is of an optical nature, it is, therefore, natural to deal with it at the image level. Moreover, several properties are only present at 


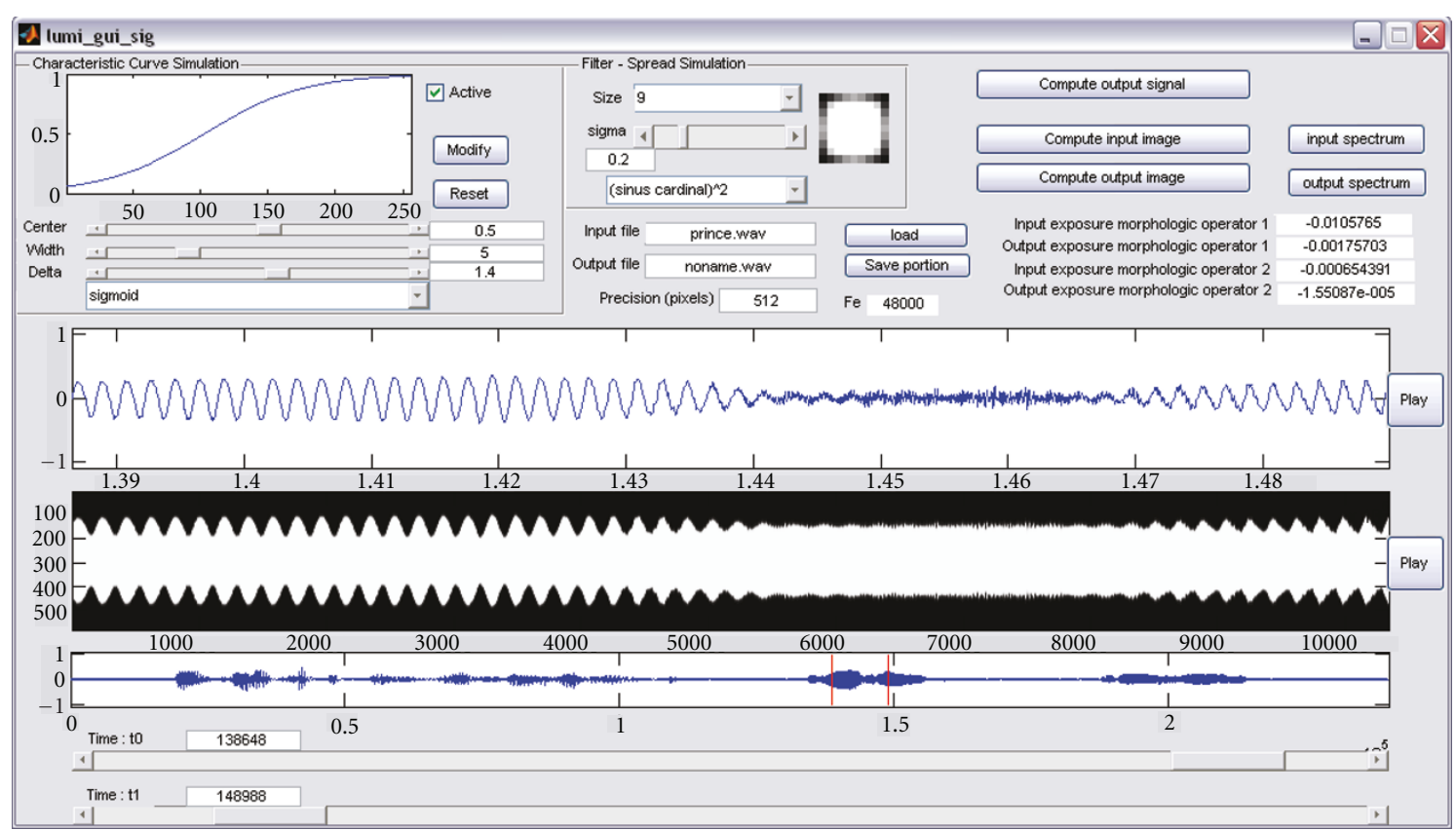

FIGURE 9: MATLAB user interface of the simulation framework. We are able to load a WAVE sound, convert it into its optical representation, simulate the image spread, and convert the signal back to WAVE. The user may set the width of the image spread function, as well as the exposure condition.

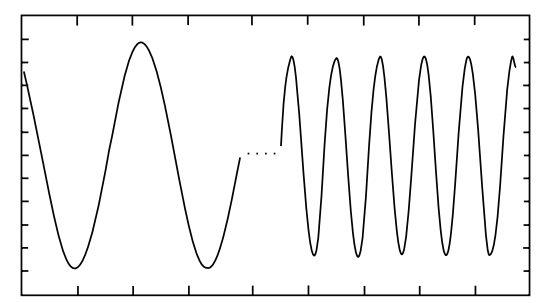

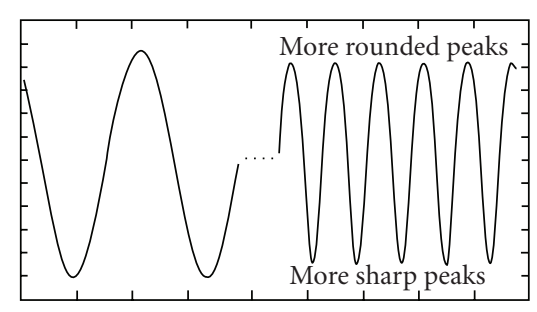

(a)

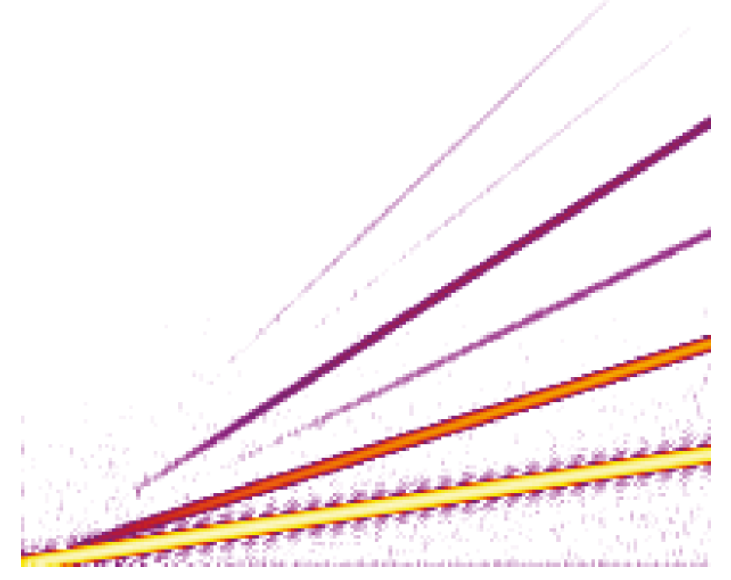

(b)

FIgURE 10: Top left: unaltered sine frequency sweep. Bottom left: altered sine sweep. The distortion introduced by incorrect exposure is noticeable at high frequency. Right: spectrogram of the beginning of the sweep. The even-order harmonics due to the desymmetrization appear first, then the odd-order harmonics caused by the change in shape.

the optical representation of the soundtrack and are lost after the conversion into an audio signal. For example,

(1) the duality object/background is not carried towards the audio signal; this point is important if the process should discriminate overexposure from underexposure;

(2) losing the gray-level transition invalidates the use of the gray-level extension of mathematical morphology operators;
(3) at last, for our experiments, we use here a really simple correction which is image based by nature, described in Section 5.

It is interesting to note that the effect of the overexposition of a soundtrack seems to be similar to the effect of the application of a morphological dilation with a certain structuring element. According to mathematical morphology theory, if this hypothesis is true, then the soundtrack should be invariant to the application of a morphological 


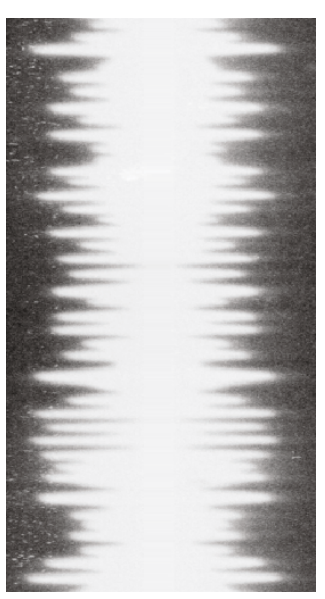

(a)

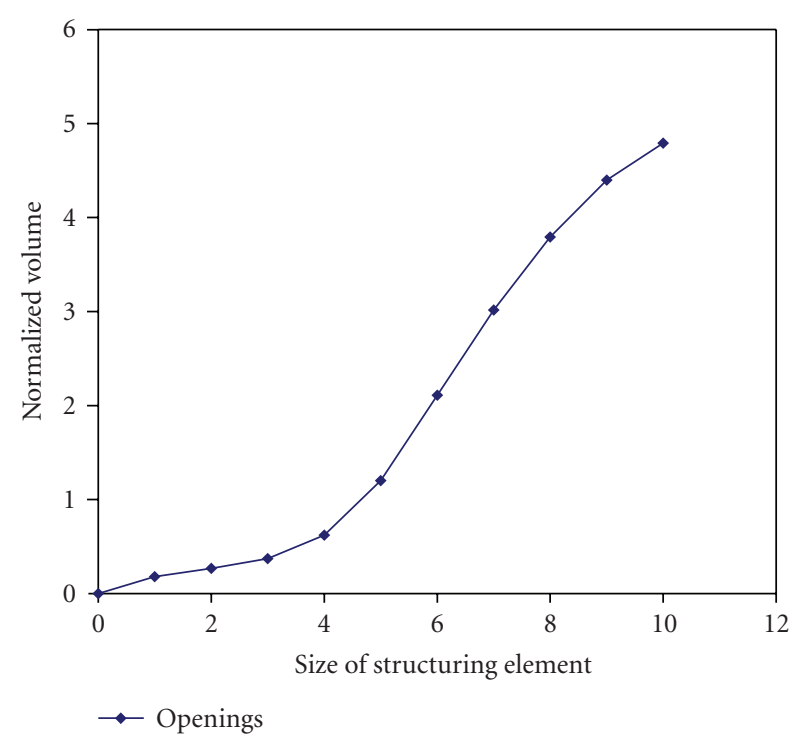

(b)

FIGURE 11: (a): overexposed soundtrack. (b): the corresponding graph: size of structuring element versus normalized volume (sum of gray values) of the difference between the original image and its successive openings.
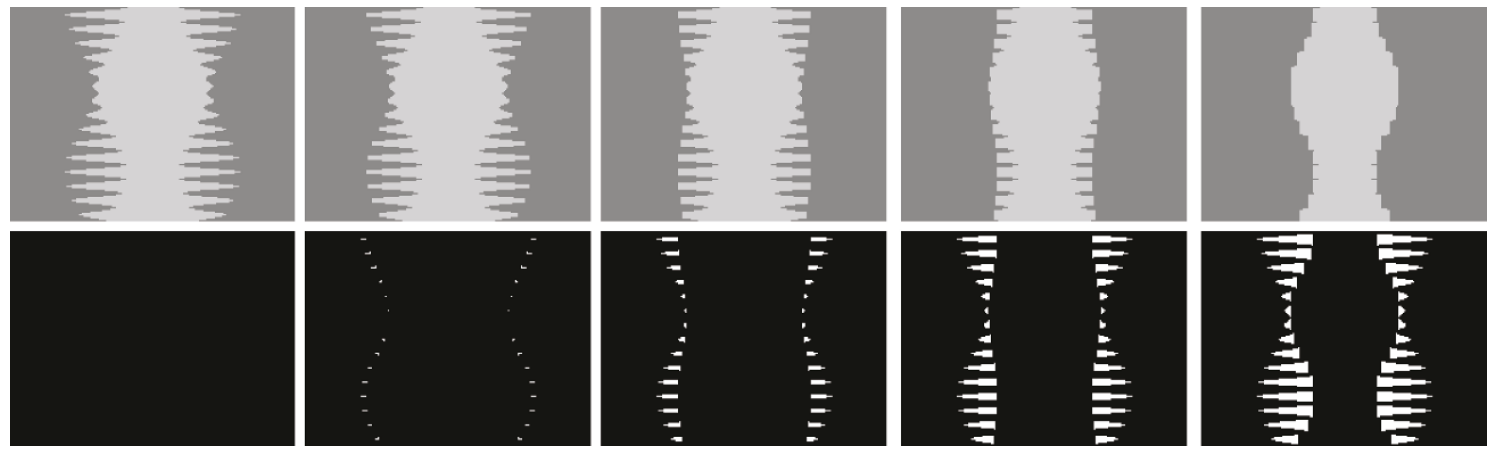

FIGURE 12: Succession of openings with vertical structuring elements and the corresponding differences (between the original image and the openings).

opening with the same structuring element. The structuring element is a priori unknown. Given the physical process that causes overexposure, it can be safely supposed that it is a disk. Several sizes (limited by the discrete nature of the scanned soundtrack) should then be tested. However, we can anticipate that the presence of noise (film grain, dust, etc.) might interfere in the verification of the hypothesis.

Therefore, we have preprocessed the image of the soundtrack using the method introduced by Brun et al. [12] in order to binarize it and suppress the noise. The application of a series of openings with structuring elements of increasing sizes allows us to check the invariance conjecture. Note that in the case of soundtracks only containing low-frequency signals, the invariance is always observed, given that such tracks do not contain thin structures, whose shape is subject to variations when overexposed. If a different behavior exists, it can only be observed in the case of high-frequency signals. In such cases, we have indeed observed a nearinvariance through a morphological opening, which tends to confirm our hypothesis (see Figure 11). The detection of underexposed soundtracks can be done in exactly the same way, by previously inverting the binary image of the soundtrack.

A second important feature is that in over-/underexposed images, the peaks and the valleys have different shapes. The peaks are sharp and the valleys are hollow or vice versa. This dissymmetry leads to the fact that the surface of the peaks is different from that of the valleys. The surface of the peaks corresponds to the volume of the difference between the original image and the succession of its morphological closings with vertical structuring elements of increasing sizes. Similarly, the surface of the valleys corresponds to the volume of the difference between the original image and the succession of its morphological openings with vertical structuring elements. To illustrate this fact, Figure 12 (resp., Figure 13) shows the succession of openings (resp., closings) with vertical structuring elements of increasing sizes applied to a soundtrack. 

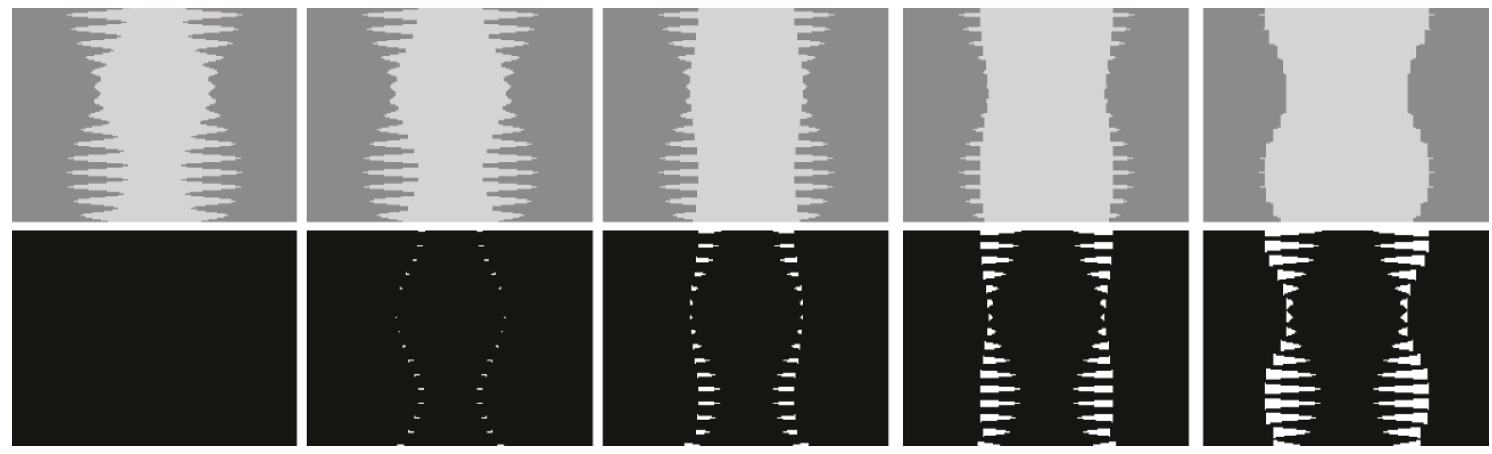

FIGURE 13: Succession of closings with vertical structuring elements and the corresponding differences (between the original image and the closings).

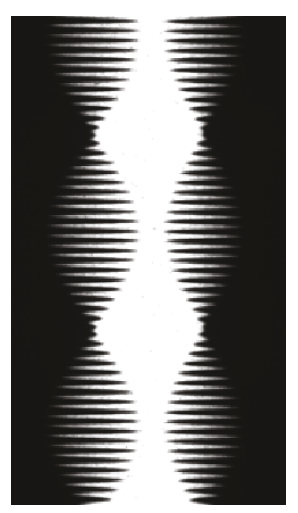

(a)

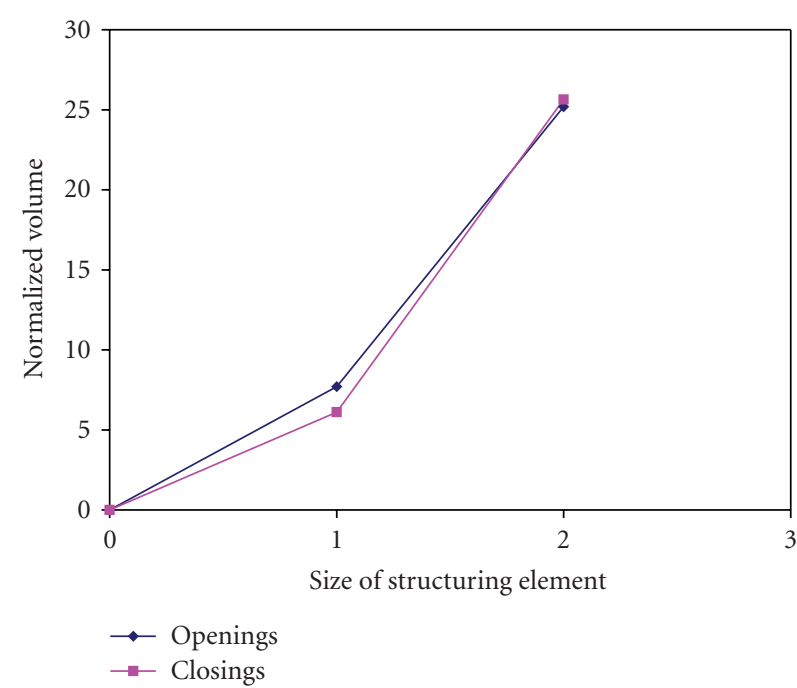

(b)

FIGURE 14: Succession of openings and closings with vertical structuring elements applied to an underexposed soundtrack.

As previously done, we have computed those successions on our images to obtain the volume of the difference between the original image and its opening (or closing) in function of the size of structuring elements. A divergence between the graph of openings and the one of closings means that the surface of the peaks is different from that of the valleys and, therefore, a bad exposure.

Figures 14, 15, and 16 show these two graphs for an underexposed, an overexposed, and a correctly exposed soundtrack. Notice that, in case of underexposure, the openings graph is located above the closings one, because the peaks surface is larger than the valleys one. The inverse phenomenon is observed in case of underexposure because the surface of the valleys becomes larger than the one of the peaks. Finally, because these two surfaces are equal in the correctly exposed soundtrack, the two graphs are nearly the same.

Once overexposure has been diagnosed, a correction is necessary. This could also be done in the image domain using mathematical morphology. In fact, we have seen that the detection of the overexposure also produces the size of the structuring element undergoing in the dilation which models the overexposure. It will be seen in Section 5.1 how this can be done.

Only severe under-/overexposition can be discerned by looking at the optical representation, and only if some reasonably high-frequency tone is present in the signal. The grabbed picture shown in Figure 8 shows such oversharp peaks. This is an extreme case, and for our project, more gentle distortions should be detected as well. Therefore, we setup two separate paths in our research planning: one approach will deal exclusively with the optical representation of the soundtrack, the second one, described here, will perform the detection step based onto the audio signal.

\section{MEASURING THE DISTORTION IN 1D AUDIO SIGNAL WITHOUT A PRIORI KNOWLEDGE}

As the $1 \mathrm{D}$ signal is more or less the transcript of the 2D VA modulation, a morphological study of the 1D signal shape will of course make sense, using, for instance, morphological operators or analysis of local derivatives of the signal. 


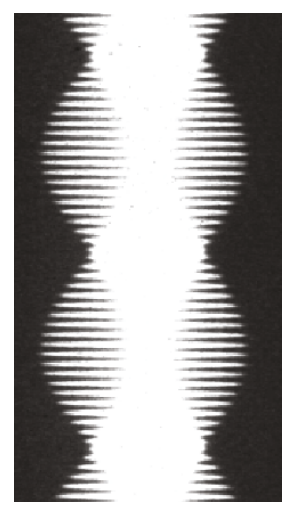

(a)

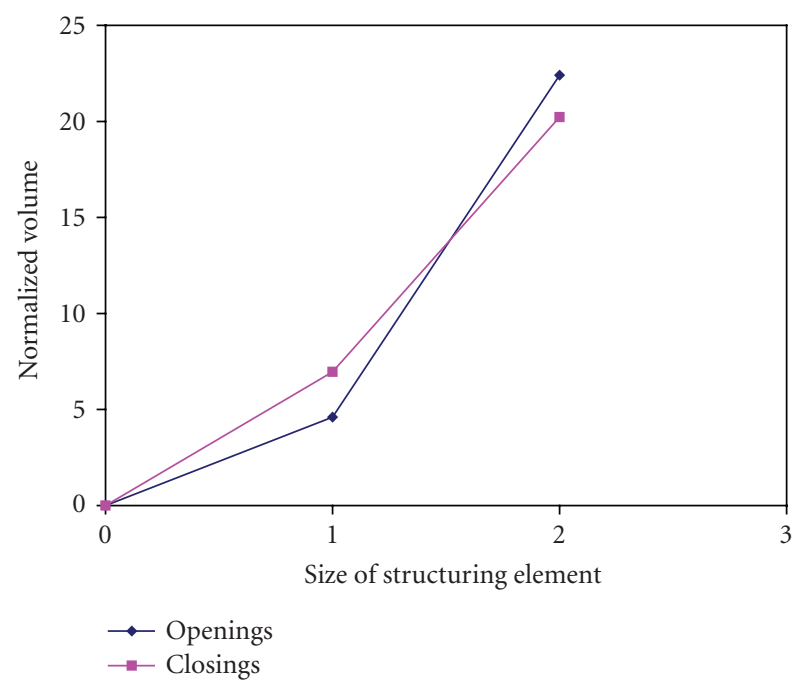

(b)

FIGURE 15: Succession of openings and closings with vertical structuring elements applied to an overexposed soundtrack.

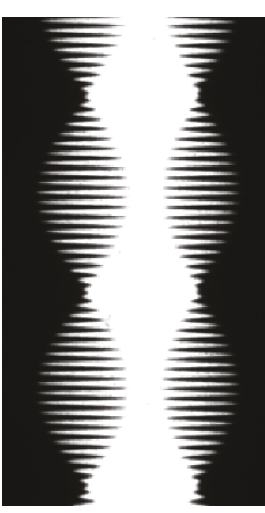

(a)

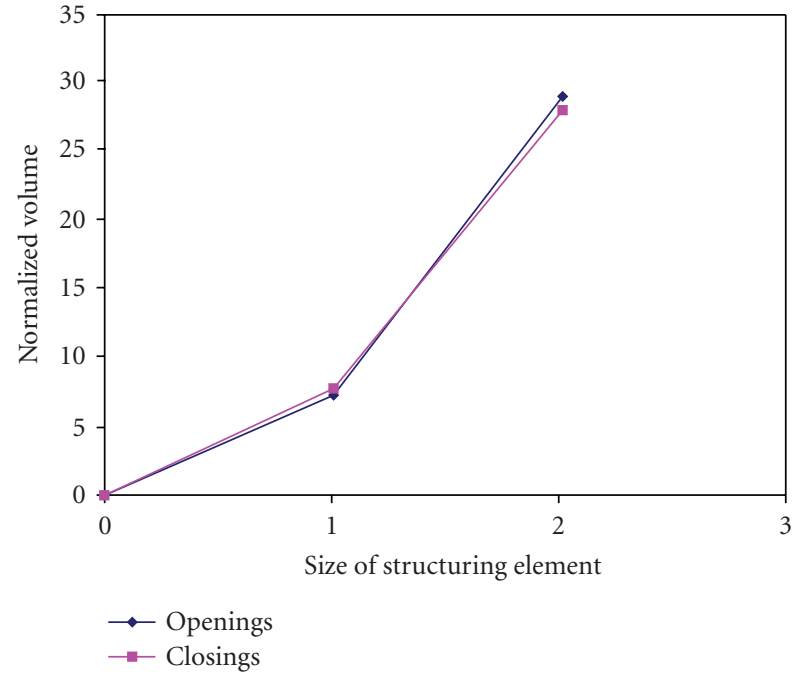

(b)

FIGURE 16: Succession of openings and closings with vertical structuring elements applied to a correctly exposed soundtrack.

Closely related to $2 \mathrm{D}$ image processing, this investigation is also conducted by Centre de Morphologie Mathématique (CMM) team.

As stated before, we focus here on the use of $1 \mathrm{D}$ audio signal for the detection and measurement of the distortion, without reference tone. Motivations are to put other techniques to work, like frequency analysis and classical signal processing, to achieve similar results. The correction itself still takes place in the $2 \mathrm{D}$ image representation of the soundtrack.

We aimed the research toward an indicator able to determine whether or not a sound sample was distorted due to incorrect exposure. Since the distortion is frequency dependant and the recorded sound can be of any nature (speech, music, etc.), composing a reliable indicator able to characterize, in an absolute manner, the magnitude of this distortion seems unrealistic. Therefore, we focused on a less robust indicator and use it in an iterative process (Figure 17). The control process operates using the variation of this indicator (between two iterations) rather than the instantaneous value of this indicator. This iterative approach should stop if the variation drops below a defined level; the amount of iteration is also restricted by the correction algorithm we use.

Usually, distortion is expressed in relation to a reference signal. So we first looked for pitch detection to automatically extract a reference, but we rapidly noticed that this will be impossible, especially for music. After discarding other methods (autocorrelation, AMDF [14]), we propose in this contribution two possible approaches. 


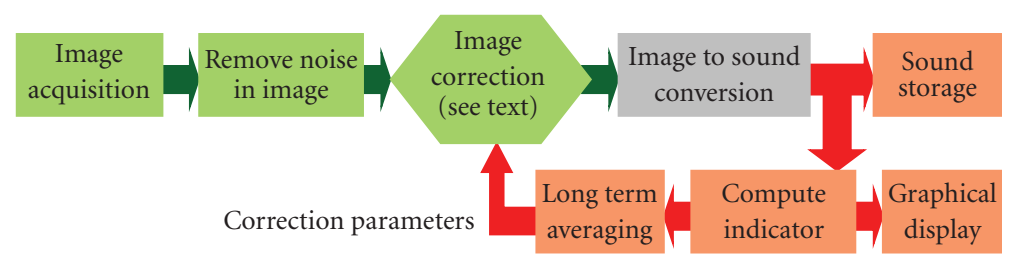

FIgURE 17: Closed-loop process.

\section{Spectrum-based indicator}

As an incorrect exposure introduces more harmonics for the higher frequencies, one of the considered approaches was to compute the center of gravity (COG) of spectrum, not only for the whole spectrum, but piecewise for different frequency ranges, and to characterize the COG shifts.

\section{Harmonic distortion-based indicator}

This indicator should reflect the harmonic distortion (mainly even harmonics) for supposed fundamental frequencies, if present.

\subsection{Distortion detection by center of gravity shifts}

The center of gravity of a spectrum (COG) is in a sense, the "mean" frequency, and this method is used for pitch detection and for audio restoration [15]. It is calculated by

$$
\operatorname{cog}(v)= \begin{cases}0, & \text { if } \sum_{n=1}^{N} v(n)=0, \\ \frac{\sum_{n=1}^{N} v(n) \times n}{\sum_{n=1}^{N} v(n)}, & \text { else, }\end{cases}
$$

where $v$ is the output vector (amplitude) from the windowed DFT at time $t$. Further, we will use the notation $\operatorname{cog}(t)$.

We compute the COG for different ranges, increasing the amount of high frequencies in the calculation. So we expect seeing the curves drifting apart if distortion is present. The COG-shift, which intends to reflect the importance of under-/overexposure, is computed by summing the distance between all possible couples of the $K$ COG as

$$
\text { COG-shift } K(t)=\sum_{n=1}^{K}\left(\sum_{l=n+1}^{K}|\operatorname{cog}(t, n)-\operatorname{cog}(t, l)|\right) .
$$

Thus, the method consists in the following steps.

(1) Compute DFT on the signal after removing impulsive noise in the $2 \mathrm{D}$ image representation,

(2) Compute COG over $K$ different ranges of the output spectrum: $[0 \ldots 1 \mathrm{kHz}][0 \ldots 2 \mathrm{kHz}] \ldots[0 \ldots 6 \mathrm{kHz}]$ $[0 \ldots 12 \mathrm{KHz}]$, therefore, $\operatorname{cog}(t, k)$ is the COG that has been computed at time $t$ of the signal for the restricted frequency range $k$,

(3) Compute COG-shift by summing distances between COG results.
Figures 18 and 19 show this behavior. We use our frequency sweep signal to illustrate the response.

Remark that the COG is related to the spectral slope. For voice (especially sonorants), the amplitude of the harmonics falls off $12 \mathrm{~dB}$ per octave or more. The shape of this plot is called the spectral slope. A flatter spectral slope, say around $6 \mathrm{~dB}$ /octave, results in stronger high frequencies, which yield a more "brassy" or strident sound. The steeper the slope, the lower is the COG. Incorrect exposure of optical soundtrack introduces harmonics and leads to a more flat plot, therefore, could also be used as an indicator.

As COG is one of many known techniques for pitch detection, the ensued indicator somehow follows the pitch of the sound sample. To be used as feedback value in our closed-loop approach, a low-pass filtering/averaging has to be applied to this value. This is not a problem, as under-/overexposure effect is constant over a long period (a complete reel, or at least over a shoot, if there are several parts spliced together on the reel).

Note that noise disturbs this method, especially impulsive noise which creates high frequencies, thus rise the COG. Fortuitously, impulsive noise is easy to remove in the image domain (dust busting).

\subsection{Harmonic distortion approach}

Total harmonic distortion (THD) is often used to characterize audio equipment, for example, amplifiers. The main cause of distortion in amplifiers is the nonlinear behavior of the gain devices (tubes and transistors) which are part of the circuit. Experienced audio engineers know that tube amplifiers often introduces even-order harmonics due to nonsymmetrical characteristics, and that class-AB amplifier introduces odd-order harmonics, du to zero crossing and clipping. This distortion depends on frequency and output power.

Several THD measures exist, among which the global total harmonic distortion (THD-G) expresses the power of a distortion in the signal.

THD-G $f$ is the THD-G for the fundamental frequency $f$ :

$$
\mathrm{THD}_{\mathrm{G}}(\mathrm{S})=\frac{\sum P_{H k}}{P_{S}},
$$

where $P_{H k}$ is the power of the $k$ th harmonic of the fundamental frequency $f$, and $P_{S}$ is the power of the input signal $S$.

The analogy to our problem (desymmetrization, clipping) is great enough to undergo a trial; but THD is 


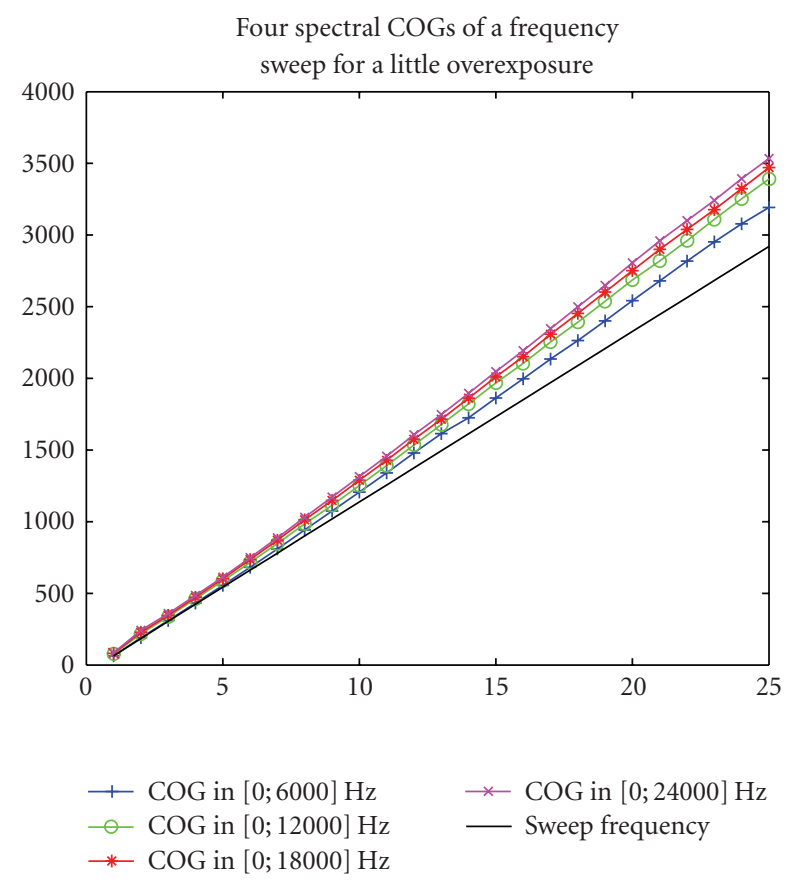

(a)

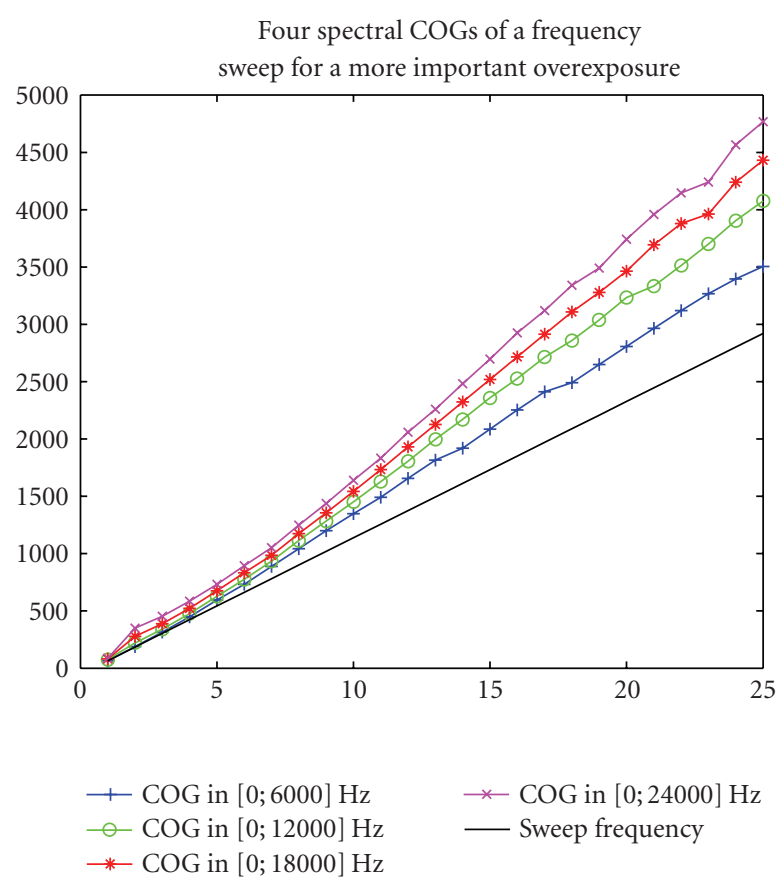

(b)

FIGURE 18: (a): COG calculation on the slightly altered sine sweep. All COG plots follow the fundamental frequency. (b): COG calculation on the sine sweep after simulation of a bad exposure. As expected, the raise of harmonics at increasing frequency shifts the COG to higher values.

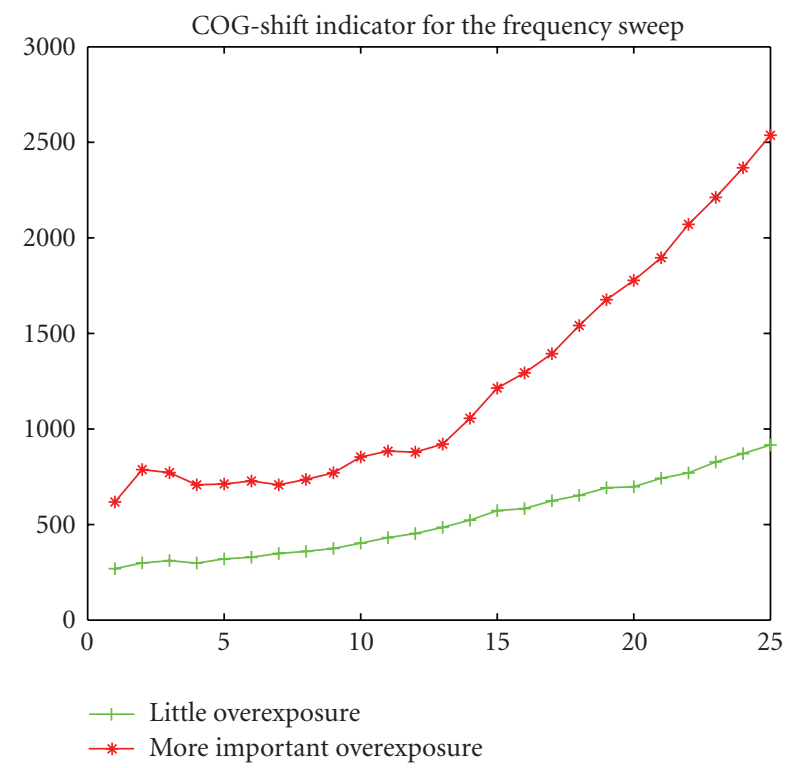

(a)
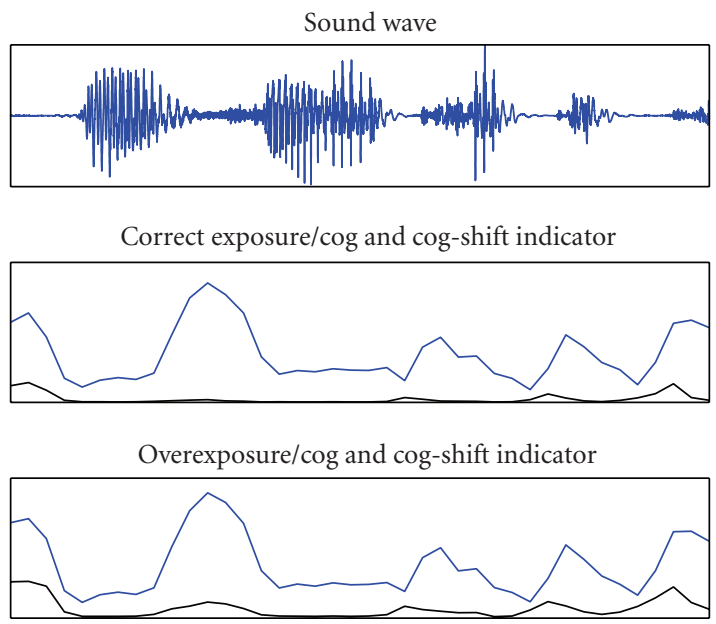

(b)

FIGURE 19: (a): COG-shift plotted over time for the frequency-sweep input. As expected, our indicator rises as frequency increase. (b): COG plot (blue) and COG-shift indicator (black) for a real-sound sample. Even if the variation is small, it is effective over the complete sample.

measured by feeding the equipment with a fixed and known signal. Measurement is reiterated for varying frequency and ends with the plot of THD versus input frequency. Since our signal is recorded without any reference, we thought about estimating (pitch detection) and measure distortion relative to it. There are several methods for pitch detection in literature, but many of these approaches are convenient to isolate a sine wave from heavy noise, but lot of methods fail for multitonal music, for example. Because of that, and inspired by [16], we investigate an ad hoc harmonic 

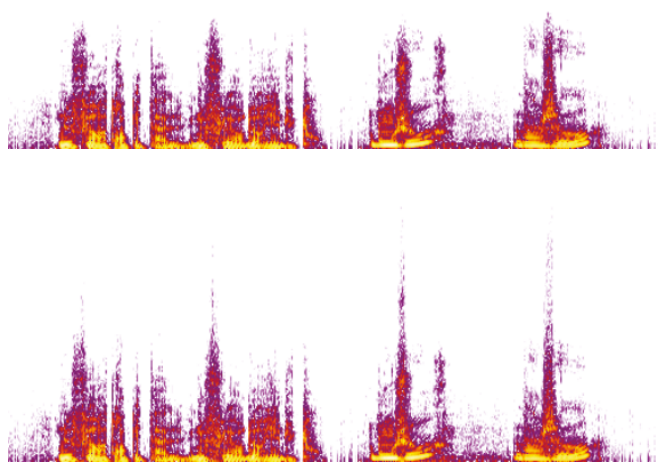

(a)

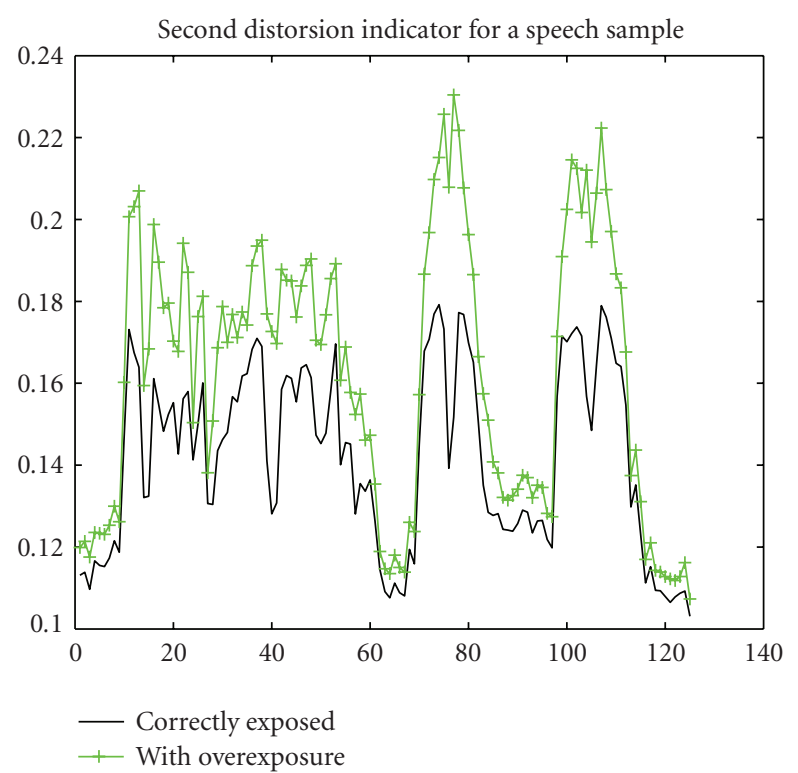

(b)

FIGURE 20: Top left: spectrogram of speech sample (5 seconds), correctly exposed. Bottom left: spectrogram of the same sample after simulation of overexposure. Right: for this sound sample, the HD-indicator is plotted in black for a correctly exposed soundtrack and in green for a overexposed one.

distortion indicator. Of course, this indicator will rise for brass music and get lower for voice, for example, but it has to reflect the change due to bad exposure for both sounds.

Consequently, our approach consists in the following steps: the input signal is filtered with a filter bank. Each filter selects one supposed fundamental frequency. For each one we compute the energy of its odd and even harmonics up to the cutoff acquisition frequency (half the sampling frequency), using two comb filters for this selection.

For the next equations, we will use the following notations:

(1) $s(t):$ is the value of $s$ at discrete time $t$, with $s(t) \in$ $[-1 ; 1]$,

(2) $s\left(t_{0}, t_{n}\right)$ : are the values of $s$ extracted ranging from $t_{0}$ to $t_{n}$,

(3) $s_{f}\left(t_{0}, t_{n}\right)$ : is the bandpass filtered (centered at $f$ ) signal (used to extract the supposed fundamental frequency $f$ ),

(4) $s_{h(f)}\left(t_{0}, t_{n}\right)$ : is the high-pass-filtered (cutoff $1.5 f$ ) signal given by $s_{\mathrm{comb}(f)}\left(t_{0}, t_{n}\right)$, where $s_{\mathrm{comb}(f)}\left(t_{0}, t_{n}\right)$ is the filtered output of $s\left(t_{0}, t_{n}\right)$ by the comb filter selecting the harmonics of $f$;

power of fundamental frequency (FP):

$$
\operatorname{FP}_{f}\left(s\left(t_{0}, t_{n}\right)\right)=\text { power }\left(s_{f}\left(t_{0}, t_{n}\right)\right)
$$

harmonics power (HP):

$$
\operatorname{HP}_{f}\left(s\left(t_{0}, t_{n}\right)\right)=\text { power }\left(s_{h(f)}\left(t_{0}, t_{n}\right)\right) ;
$$

and the power function is

$$
\text { power }\left(s\left(t_{0}, t_{n}\right)\right)=\frac{1}{t_{n}-t_{0}+1} \sum_{t=t_{0}}^{t_{n}}(s(t))^{2} \text {. }
$$

These supposed fundamental frequencies have been arbitrarily chosen, keeping in mind a future fast IIR implementation. Moreover, for easy-comb filter design, the rule $2 \times f_{s}=$ $f_{e} / n$ should be applied ( $f_{s}$ the sampling frequency, $f_{e}$ the supposed fundamental frequency, and $\left.n \in I N^{*}\right)$. Our set contains the following frequencies (in Hz): 192240480750 1200160020003000400048006000 . Filter design for both bandpass filters and comb filters has been done thanks to MATLAB's filter design tool.

We plot these "harmonic distortion" values against time for several signals (frequency sweep, voiced signal, music) before and after alteration by our simulator, we combined the results in order to find an indicator which reflects the distortion introduced by a faulty exposure (see Figures 20 and 21).

Harmonic Distortion Indicator $\mathrm{HD}$ is null when power $\left(s\left(t_{0}, t_{n}\right)\right)=0$, else it is expressed as follow:

$$
\begin{aligned}
& \text { HD-indicator }\left(s\left(t_{0}, t_{n}\right)\right) \\
& =\left(\log _{10}\left(\frac{1}{\text { power }\left(s\left(t_{0}, t_{n}\right)\right)} \sum_{f \in \text { filterbank }} \frac{\operatorname{FP}_{f}\left(s\left(t_{0}, t_{n}\right)\right)}{\operatorname{HP}_{f}\left(s\left(t_{0}, t_{n}\right)\right)}\right)\right)^{-1} .
\end{aligned}
$$

As expressed in (7), the indicator is based on the summation of the ratio $\operatorname{FP}_{f}\left(s\left(t_{0}, t_{n}\right)\right) / \mathrm{HP}_{f}\left(s\left(t_{0}, t_{n}\right)\right)$ for all $f_{e} \in$ filterbank. To avoid high values for signal parts with little modulation (low frequencies, moments of silence), 


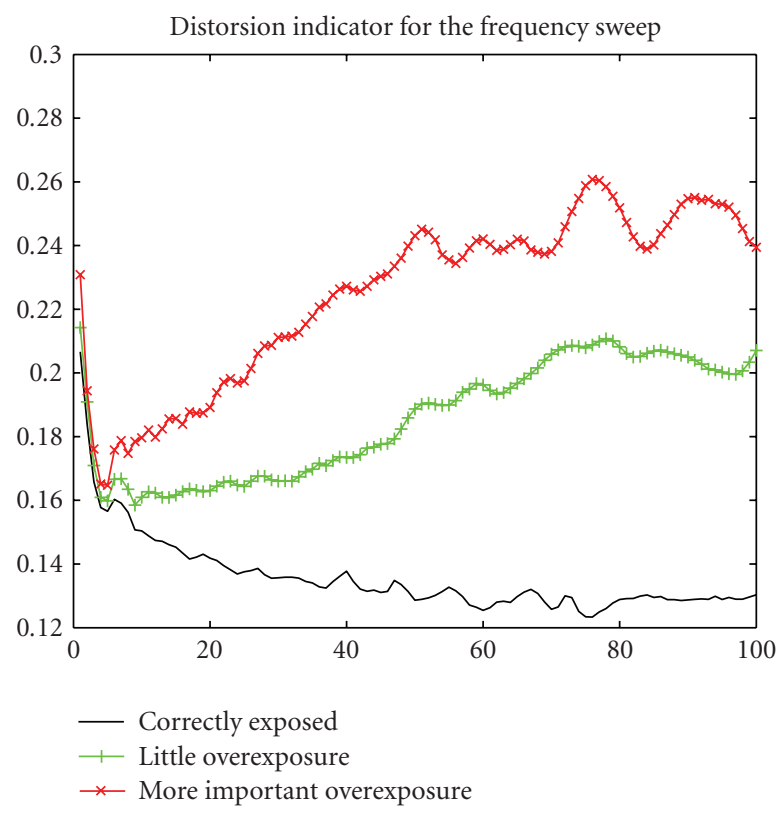

(a)

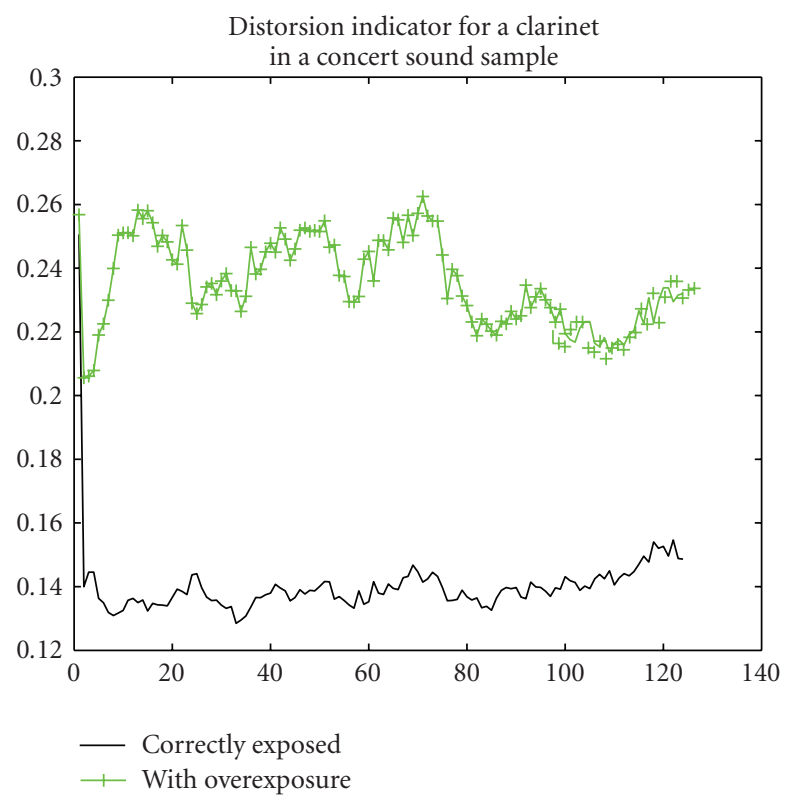

(b)

Figure 21: (a): HD-indicator for the frequency sweep test signal (black: correct exposure, green: light overexposure, red: strong overexposure). (b): HD-indicator for music instrument (clarinet) sample (black: correct exposure, green: light overexposure).

the ratio is weighted by the signal power for this part (power $\left.\left(s\left(t_{0}, t_{n}\right)\right)\right)$. Since power $\left(s\left(t_{0}, t_{n}\right)\right) \in[0 ; 1]$, the $\log 10$ scale smooths out abrupt variations. Because we want our indicator to increase with the distortion, we take the inverse of this expression.

Even if the behavior of the indicator must be deeper studied (immunity to noise, linearity, performance for moments of silence, etc.), using it in the closed-loop scheme and minimizing it while iterating gave us acceptable results (given the simple correction we used).

\section{CORRECTION OF THE 2D OPTICAL REPRESENTATION OF THE SOUNDTRACK}

A very simple correction was setup to experiment our "closed-loop" solution. For this, the images are grabbed with a great dynamic range (our line-scan camera is able to output 12 bits/pixel) together with a fine tuning of lightning power and camera integration time. Consequently, we are able to change the intensity levels of the image pixels over a great range. For test purposes, we also optically blur the soundtrack (defocussing the camera). This cuts the bandwidth, but also enlarges the blending area from black to white; therefore, the suggested correction is more efficient.

The high dynamic range image is mapped to an 8bits/pixel image by following these rules.

(1) The histogram of the 12 bits/pixel image is computed. The two peaks are detected (corresponding to soundtrack and surroundings). These grey-levels $p_{\min }$ and $p_{\max }$ are used for the subsequent steps.

(2) A second tone mapping is performed, in form of a histogram stretching directed by the indicator. The feedback sign is manually set, since the distortion detection in the audio signal does not differentiate overexposure from underexposure. For this histogram stretching, the new maximum value (resp., minimum, according to feedback sign) is decreased (resp., increased) by a value ( $c_{p}$. indicator) where $c_{p}$ is experimentally set (a complete proportionalintegral-derivative control at each iteration should perform better, assuming indicator smoothing as well). The output is shown in Figure 22. The process is reiterated and stopped after a fixed amount of iterations or if indicator drops below a threshold. If the amount of iterations is not restricted, the correction itself stops if minimum reaches (maximum -1 ) (resp., maximum reached $($ minimum +1$)$ ), releasing hence a binary image.

This simple correction, intended as a proof of concept, makes use of the image spread (present at photographic level, emphasized by the slightly blurred acquisition) and shifts the gray-levels towards black level (resp., towards white level). Obviously, as the correction is iterated, the image loses in dynamics and aliasing appears (Figure 23). On the other side, this kind of correction is really fast (using Look-Up tables).

$I_{\text {in }}$ : pixel of the 12 bits/pixel image, as grabbed,

$I_{\text {out }}$ : pixel of a 8 bits/pixel image, used for indicator calculation,

$c_{p}$ : coefficient for the proportionnal term of the regulation loop,

if overexposure: $\quad p_{\min }=p_{\min }+\left(c_{p}\right.$. indicator $)$,

if underexposure: $p_{\max }=p_{\max }-\left(c_{p} \cdot\right.$ indicator $)$

$I_{\text {out }}=\left(I_{\text {in }}-p_{\min }\right) \frac{b-a}{p_{\max }-p_{\min }}+a$. 


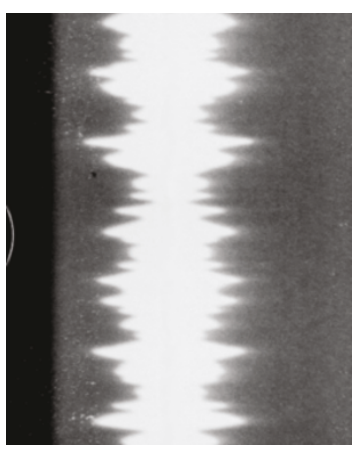

(a)

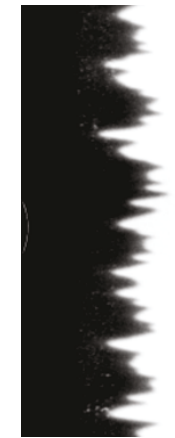

(b)

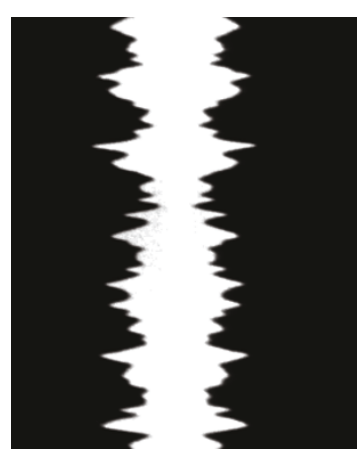

(c)

Figure 22: Optical representation of ca. 1/75 second of sound from the "L'acrobate" soundtrack. (a): as grabbed, Middle: histogram stretching at first iteration, between $p_{\min }$ and $p_{\max },(\mathrm{b})$ : after several iterations according to indicator minimization.
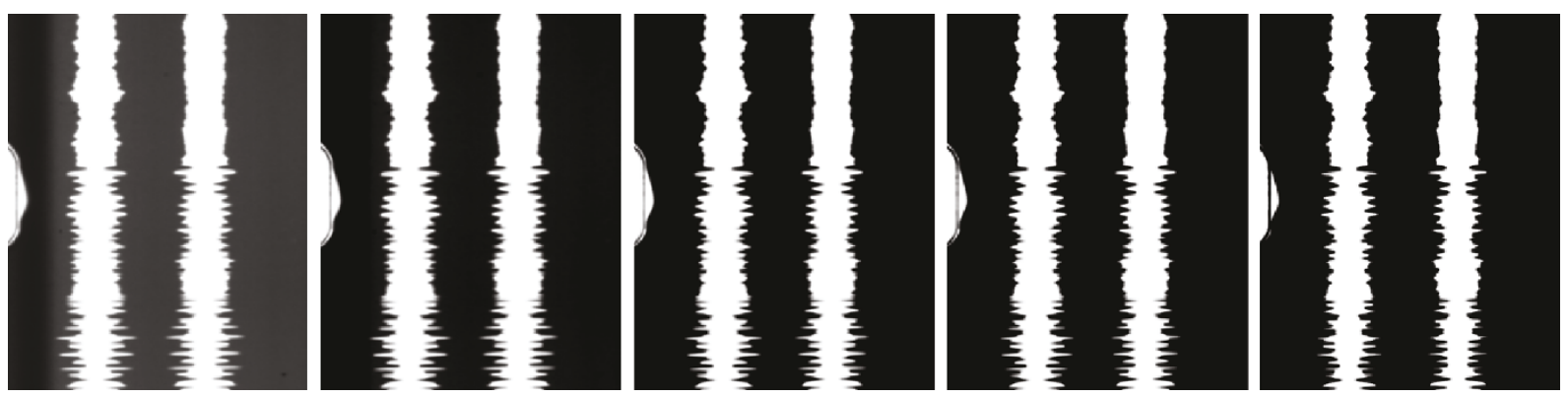

(a)

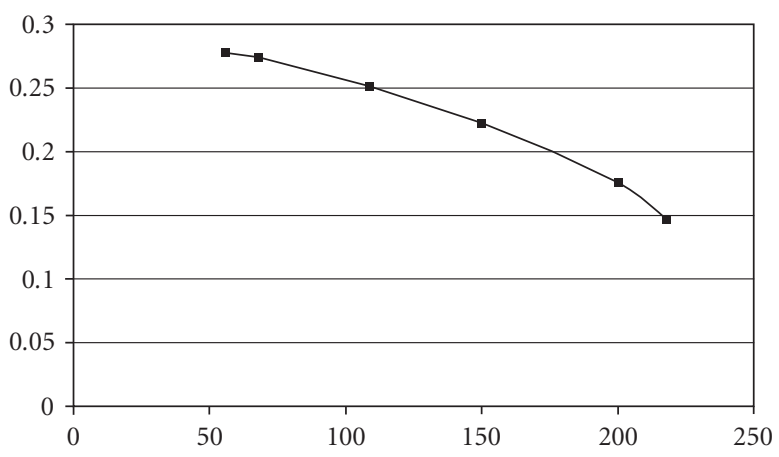

(b)

FIGURE 23: Optical representation of ca. 1/75 second of stereo soundtrack. From left to right: as grabbed, histogram stretching at first iteration (based on histogram), 2nd, 3th, and 4th iteration. Below: plot of the HD indicator value versus $p_{\min }$. The HD indicator values for this plot is the mean value computed on 64000 samples (1,33 seconds).

\subsection{Correction by mathematical morphology}

Considering real data, especially the "L'acrobate" soundtrack (opening credits music from the movie "L'acrobate" (1940)), the visual examination of the acquired images advise us that a simple correction based on a transfer function should not be sufficient.

We have supposed in Section 3 that overexposure can be modelled as a morphological dilation, and we have explained how to validate this hypothesis and compute the size of the corresponding structuring element. If this hypothesis is true, then the theory of mathematical morphology tells us that some information might have been lost in the process, and that a good candidate for the restoration is obtained with a morphological erosion using the same structuring element. Underexposed soundtracks would be restored analogously by using a dilation.

\section{CONCLUSION AND FORTHCOMING WORK}

Validation has been performed on simulated data but also on real data, but for the latter, we do not hold any unaltered 


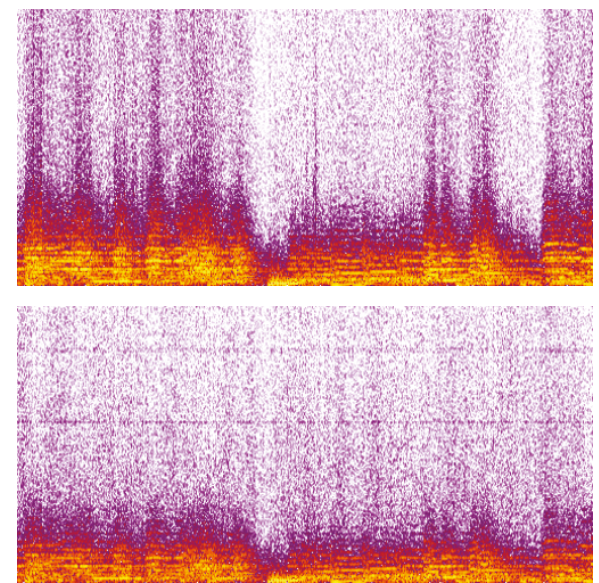

(a)

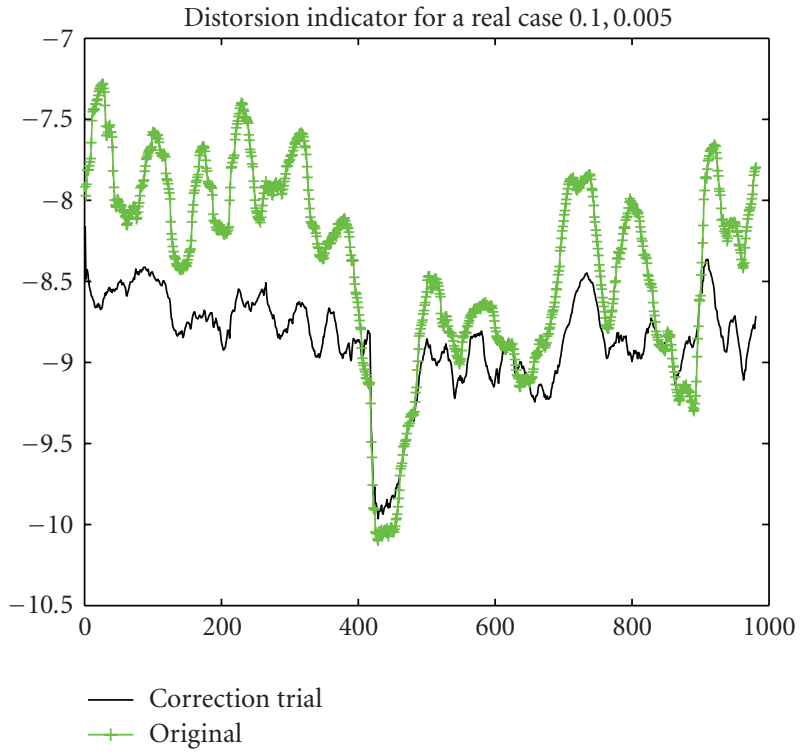

(b)

Figure 24: Top left: spectrogram of real soundtrack (“L'acrobate," 5 seconds), grabbed by our scanner and converted to sound. Bottom left: spectrogram of the same sample after correction. Notice the noise level for real soundtracks (here no dust removal was performed). Right: for this sound sample, the HD-indicator is plotted in green before correction and in black after correction.

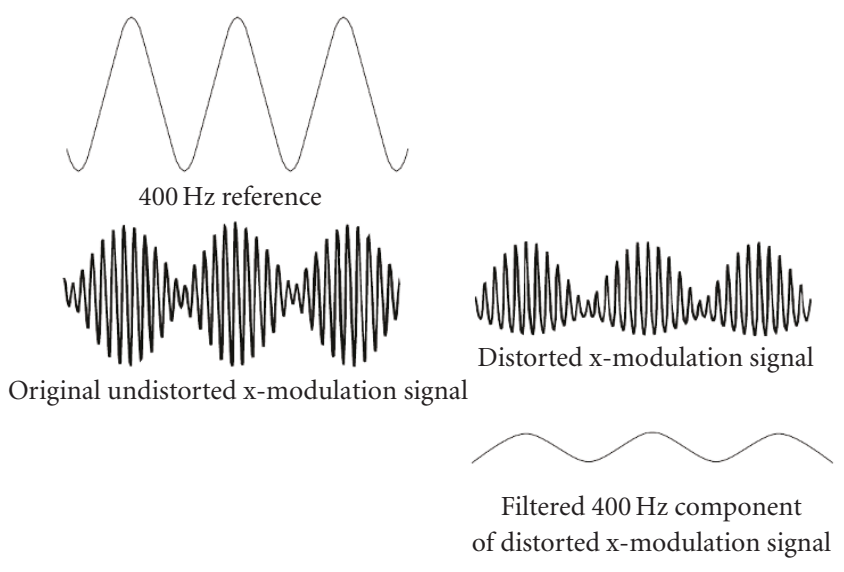

(a)

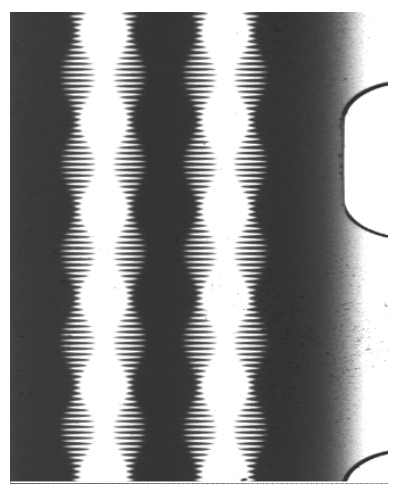

(b)

FIGURE 25: (a): graphical illustration of the cross-modulation test (lifted from Kodak's technical note "cross-modulation distortion testing for the motion picture laboratory"). (b): image grabbed from a real cross-modulation test reel (stereo tracks).

counterpart to compare with. The results look promising, to be said that it is easier at this stage to do a visual assessment of the restored images or compare spectrograms (Figure 24) rather than listening to the converted sound.

Using pure image processing for detecting this impairment involves operators which are noise sensitive, especially dust located near the "black to white" transitions. A perfect digital cleaning of the tracks is a tedious process, up to now too slow for implementation, and the related research on this process is out of the topic of this paper. Hence, our proposal to use signal processing in the audio domain for distortion detection makes sense and is easier, since the way the soundtracks are read (integration over a line) minimizes the incidence of dust.

On the contrary, using image-based correction seems to be mandatory. The simple correction scheme used for the proof of concept (adjusting the luminance distribution) is interesting because it is simple and related to the steepness of the grey-level slope in area where image spread occurs. However, for high degrees of incorrect exposure, the correction will need support of more complex operators. This will be a forthcoming work.

Both indicators seem valuable, but the COG-shift is too sensitive to noise present in moment of silence (MOS). 
Nevertheless, both indicators tend to follow the pitch, therefore, settings the rights coefficient in a PID regulation scheme and adjusting the window sizes for FFT and filtering have to be investigated.

Opening up an unmarked application field, the solution proposed is very innovative in its construction by coupling signal processing and image processing in a regulation loop. A valuable simulation framework has been set up, and some methods have been investigated to extract an indicator reflecting the distortion caused by under-/overexposure without prior knowledge. The open loop behavior of indicator $(S)$ needs to be more deeply investigated (monotony, linearity, etc.).

The presented work (computation of indicators, simple correction) is about to be coded in real time, using Intel performance primitives (IPP), as a computing stage closely coupled to the image acquisition stage of the RESONANCES soundtrack scanner.

At last, as an absolute improvement is hard to perceive while listening to a real-altered sound sample, comparative listening will be meaningful for the sound samples and their simulated degraded duplicate. Blindfold listening test at a postprocessing auditorium is planned.

\section{APPENDIX}

\section{THE CROSS-MODULATION TEST}

Soon after the introduction of optical soundtracks in the movie industry, the processing labs asked for a procedure to determine the optimum exposure conditions for both negative and print. From the forties forward, an industrystandard practice raised, commonly known as the "crossmodulation test," and is still used as a quality assurance routine prior to sound recording and duplication. The test is based on the fact that a perfect sinusoid comprising a highfrequency signal (about $10 \mathrm{kHz}$ ) modulated at $75 \%$ by a lowfrequency one (typically $400 \mathrm{~Hz}$ ) will have an average value of zero (the average light transmission will be constant). In the case of underexposure or overexposure, some of the lowfrequency modulation component will be introduced into the average value of the signal and may be detected. Figure 25 illustrates this process. A low-pass filter is connected after the optical pickup head to eliminate the high-frequency carrier, and the amount of $400 \mathrm{Hertz}$ signal remaining is analyzed to determine the exposure and printing conditions which result in the lowest-level signal. That means a technician reads a simple needle display showing the average level and graphs values against processing parameters.

This technique is still used and we suggest the eager readers to study further the technical note from Kodak [17] on the cross-modulation test.

\section{ACKNOWLEDGMENTS}

This work was made possible thanks to the financial help of the French Agence Nationale de la Recherche, through its RIAM program. The film material, as well as the expertise on motion picture optical soundtracks, were provided by $\mathrm{N}$.
Ricordel from the CNC-Archives Françaises du Film and by C. Comte from GTC-Eclair Group.

\section{REFERENCES}

[1] E. W. Kellog, "History of sound motion pictures," Journal of the SMPTE, vol. 64, pp. 291-302, 1955.

[2] J. G. Frayne and H. Wolfe, Sound Recording, John Wiley \& Sons, New York, NY, USA, 1949.

[3] Erpi ClassRoom Films Inc., Sound recording and reproduction (sound on film). An instructional sound film, 1943, http://www.archive.org/details/SoundRec1943.

[4] J. Monaco, How to Read a Film, Oxford University Press, Oxford, UK, 3rd edition, 2000.

[5] "Cinematography-A-chain frequency response for reproduction of $35 \mathrm{~mm}$ photographic sound-Reproduction characteristics," International Norm ISO 7831, 1986.

[6] P. Streule, Digital image based restoration of optical movie sound track, M.S. thesis, Electronics Labs, Swiss Federal Institute of Technology, Zurich, Switzerland, March 1999.

[7] D. Richter, D. Poetsch, and A. Kuiper, "Localization of faults in multiple double sided variable area code sound tracks on motion picture films using digital image processing," in Proceedings of the 13th International Czech - Slovak Scientific Conference Radioelektronika, Brno, Czech Republic, May 2003.

[8] D. Poetsch, D. Richter, and I.-H. Kurreck, "Restoration of optical variable density sound tracks on motion picture films by digital image processing," in Proceedings of the International Conference on Optimization of Electrical and Electronic Equipments (OPTIM '00), pp. 793-798, Brasov, Romania, May 2000.

[9] A. Kuiper and L. Dzbnek, "Localization of faults in multiple double sided variable area sound tracks on motion picture films using digital image processing," Departement of Radio Electronics, FEEC, BUT, 2005.

[10] A. Kuiper, "Detection of dirt blotches on optical soundtracks using digital image processing," in Proceedings of the 15th International Czech - Slovak Scientific Conference Radioelektronika, Brno, Czech Republic, May 2005.

[11] J. Valenzuela, "Digital audio image restoration: introducing a new approach to the reproduction and restoration of analog optical soundtracks for motion picture film," in Proceedings of the International Broadcasting Convention (IBC '03), Technicolor Creative Services, Amsterdam, The Netherlands, September 2003.

[12] E. Brun, A. Hassaine, B. Besserer, and E. Decenciere, "Restoration of variable area soundtracks," in Proceedings of the IEEE International Conference on Image Processing (ICIP '07), pp. 13-16, San Antonio, Tex, USA, September 2007.

[13] J. Serra, Image Analysis and Mathematical Morphology, vol. 1, Academic Press, London, UK, 1982.

[14] G. S. Ying, L. H. Jamieson, and C. D. Michell, "Probabilistic approach to AMDF pitch detection," in Proceedings of the 4th International Conference on Spoken Language Processing (ICSLP '96), vol. 2, pp. 1201-1204, Philadelphia, Pa, USA, October 1996.

[15] A. Czyzewski and P. Maziewski, "Some techniques for wow effect reduction," in Proceedings of the IEEE International Conference on Image Processing (ICIP '07), vol. 4, pp. 29-32, San Antonio, Tex, USA, September 2007. 
[16] R. A. Irizarry, "Local harmonic estimation in musical sound signals," Journal of the American Statistical Association, vol. 96, no. 454, pp. 357-367, 2001.

[17] "Cross-modulation distortion testing for the motion picture laboratory," Tech. Rep., Eastman Kodak Company, Rochester, NY, USA, 2001, http://www.kodak.com/US/plugins/acrobat/ en/motion/support/h44/h44.pdf. 